\title{
Acceso al ciberespacio: algunas notas y cifras
}

La tan aclamada globalización y su consorte, la informatización, ya han logrado un nivel de penetración en casi todos los rincones de la vida moderna. $\mathrm{Si}$ bien se genera una desconfianza natural que se ha convertido en una resistencia organizada, es necesaria una profundización objetiva y crítica para evitar caer en las utopías facilonas o en los golpes de pecho perpetuos. Tenemos que responsabilizarnos de nuestro papel (como investigadores, como académicos, como estudiantes o como ciudadanos) y ésta es una labor que requiere la atención y la acción conjunta e interdisciplinaria que involucre a expertos en derecho, comunicación, tecnologías, políticas de desarrollo y sociedad civil, para lograr con ello un entendimiento más cercano del fenómeno y así poder actuar en consecuencia.

No podemos negar que la informatización está cambiando la manera de vivir de la sociedad moderna ya que:

Las tecnologías de la información y la comunicación tienen la capacidad de transformar profundamente los modos en que la gente organiza su vida, interactúa con otras personas y participa en los diversos ámbitos de la sociedad. Estas tecnologías son los cimientos de un cambio radical que, desde las definiciones industriales/postindustriales de desarrollo, dará origen a un nuevo paradigma basado en el modelo de las sociedades de la información.

Una de las preocupaciones constantes y cuestión central en la discusión sobre la llamada "Sociedad o Era de la Información" es la referente al acceso a la información.

1. Facultad de Letras y Comunicación. Universidad de Colima. 
A finales del siglo y de milenio, el derecho a saber de los ciudadanos ha adquirido un valor significativo, circunstancia que se refleja en un ciudadano más exigente, que inquiere y cuestiona, interesado sobre todo en contar con los mayores elementos de información a su alcance para ejercer de manera óptima sus derechos y cumplir con sus obligaciones, haciendo viable de esa forma la democracia posible en la sociedad de información (Villanueva, 1995, p.96-97)

Sin embargo, una discusión anterior y que muchas veces queda pendiente en las acaloradas discusiones sobre globalización y acceso a la información es la que plantea la interrogante sobre el acceso a las herramientas o medios para llegar a dicha información, en el entendido que:

El desarrollo de las nuevas tecnologías de comunicación puede ser abordado desde la perspectiva que intenta explicar a la tecnología como un elemento que define no sólo la forma en que se producen y circulan los mensajes, sino como un elemento constitutivo de los procesos de comunicación en tanto que procesos de producción y apropiación de bienes simbólicos (Spíndola, 1995, p. 173).

En este trabajo pretendemos ligar algunos datos y elementos teóricos que nos ayuden a entender la situación actual con respecto a las nuevas tecnologías de la comunicación, en específico el Internet, y continuar la oportuna reflexión a la luz del acceso a la información como elemento clave en la constitución de una sociedad democrática. Porque "mientras el derecho a la información habilita para obtener noticias, la libertad de expresión y de imprenta facultan a difundirlas, de ahí que sean derechos complementarios" (Villanueva, 1995, p.97).

\section{El acceso al ciberespacio; algunas cifras}

El número de usuarios de Internet alrededor del mundo está en constante crecimiento, siendo éste casi exponencial. El Computer Industry Almanac ${ }^{2}$ reportó que para el año 2002, 490 millones de personas alrededor del mundo tendrán acceso a Internet, esto es, 79.4 por cada 1,000 personas, y 118 por cada 1,000 para el final del año 2005. Solamente quince países, los más importantes en esta acelerada carrera de acceso a Internet, tendrán el $82 \%$ de usuarios (incluyendo usuarios para negocios, educación y hogar). Los Estados Unidos son el país con mayor porcentaje de acceso y ejercen un liderazgo aplastante con más de 110 millones de usuarios proyectados al final de 1999 , lo que representa casi el $43 \%$ de un total de 259 millones de usuarios de

2. Almanaque de la Industria de la Computación. 
Internet. Actualmente el $42 \%$ de los hogares hispanos en Estados Unidos poseen una computadora y mientras que la penetración de computadoras en los hogares estadounidenses ha crecido en un $43 \%$ en los últimos dos años, en el caso concreto de los hispanos el crecimiento ha sido del $68 \%$. Sin embargo, contrariamente a lo que se pudiera pensar al hablar del acceso a Internet en países desarrollados como Estados Unidos, las cifras demuestran que, no obstante la penetración tan grande en los hogares, escuelas y lugares públicos, sigue habiendo una clara y amplia brecha entre las personas que tienen acceso y las que no. Por ello, se están tomando medidas el respecto; un reporte del grupo Gartner ${ }^{3}$ titulado "División digital y sociedad estadounidense" encontró que la mitad de los hogares americanos tienen acceso a Internet y para el 2005 , esta cifra llegará al $75 \%$. Aun así, recomiendan mayor participación gubernamental para acortar esta brecha entre los que tienen acceso y los que no. El presidente de la compañía, Michel Fleischer, dice que una posibilidad sería con impuestos que generen créditos para que empleados puedan tener acceso a Internet. Nuevamente nos damos cuenta de que existe una política abiertamente pro-tecnológica. Ahora bien, otro estudio realizado por la "Pew Internet \& American Life Project" ${ }^{4}$ demuestra que el acceso tiene que ver más con el poder adquisitivo que con la cuestión de la raza, como se había pensado en algún momento, ya que el $50 \%$ de los blancos tienen acceso a Internet comparado con el $36 \%$ de los negros y el $44 \%$ de los hispanos. $\mathrm{Al}$ respecto autores como Barroso (1998) señalan que:

La tecnología informática podría realzar la democracia e igualar a todos en el acceso a la información, ayudar a los inválidos y a los pobres, pero estos potenciales sólo se realizarán mediante acciones deliberadas de las políticas públicas y de las elecciones personales y profesionales (p. 88)

Es decir, el Internet pudiera ser un medio que por ser extendido y horizontal (por lo menos en su estructura técnica) pudiera atenuar las actitudes y políticas racistas y clasistas. Sin embargo, la reflexión es que si en el país con mayor desarrollo tecnológico del mundo existe esta desigualdad de acceso y una inexistencia de políticas para acortarla, ¿qué podemos esperar en otros países, sobre todo de los que se encuentran en vías de desarrollo? Existe, o debería existir, una preocupación creciente por la brecha entre los que tienen acceso y los que no, no sólo por las cuestiones obvias (el comercio en Internet es la clave para los grupos que controlan el acceso) sino porque si se entra tarde en la carrera tecnológica, la participación será solamente como consumidor de mensajes, productos, ideas y tecnología creando con ello una de-

3. http://www.gartner.com

4. http://www.pewinternet.org 
pendencia total de los países poco desarrollados tecnológicamente respecto a los más desarrollados (cfr. Mosco, 1989). En realidad, este proceso que se inició con la colonización, se desarrolló con la industrialización y llegó a su punto más álgido con la economía neoliberal y global encontraría así su concreción total con la informatización. Por supuesto que por sí solos los instrumentos no podrán asegurar la realización plena de un proyecto democratizador e igualitario en la sociedad, como lo señala Hamelink (1991) al decir que "es evidente que las próximas décadas confrontarán una penetrante utilización de las técnicas de información. Sin embargo, es ilusorio y engañoso pensar que esto termine con las desigualdades sociales" (p. 18). Pero es un primer paso para este fin, ya que, continúa: "Las sociedades tendrán que elegir una conciencia diferente, formas distintas de pensamiento y conocimiento, y una cultura más receptiva, intuitiva y autónoma" (p. 26), pero esto sólo podrá lograrse si se compite en las mejores condiciones y con el equipamiento necesario, como lo apunta un reporte de la UNESCO: ${ }^{5}$

Los países del Sur no pueden seguir percibiendo las tecnologías de la información y la comunicación como un lujo exclusivamente reservado a los países desarrollados. Hoy en día, los países en desarrollo ya no deben cuestionarse sobre la conveniencia de otorgar máxima prioridad a su participación en la estructura mundial y nacional de información como sobre la forma adecuada de aplicar al desarrollo económico, sociopolítico y cultural las tecnologías de la información para reducir más que incrementar la distancia que separa a los poseedores de los desposeídos de información.

\section{América Latina, sus cifras}

La situación en América Latina es mucho más extrema. Aunque los costos de acceso a Internet han disminuido en los últimos doce meses $(23 \%, 20 \%$ y $8 \%$ en Argentina, Brasil y México respectivamente), esto no es suficiente para crear masa crítica, pues más de la mitad de la población no estará en línea en los próximos años. Claramente el Internet hispanoamericano está en franco crecimiento. Resaltamos aquella proyección que indica que en el año 20005 el $12 \%$ de la población hispanoamericana hará uso de las tecnologías de información y comunicaciones. Esta cifra es modesta y tiene directa relación 
con la falta de infraestructura, los altos costos de comunicación y la falta de capacitación y desarrollo de contenidos locales en nuestra región.

Las siguientes gráficas muestran la evolución de Internet en México ${ }^{6}$ y en América Latina ${ }^{7}$ respectivamente:

\begin{tabular}{|c|c|c|c|}
\hline Foche & Utusties & Fuente & Metodelogia \\
\hline 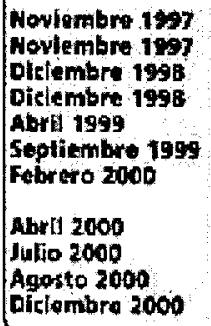 & $\begin{array}{r}596,000 \\
370,000 \\
50,900 \\
713,000 \\
600,000 \\
900,000 \\
1,300,000 \\
2,450,000 \\
2,500,000 \\
5,323,020 \\
2,938,000\end{array}$ & 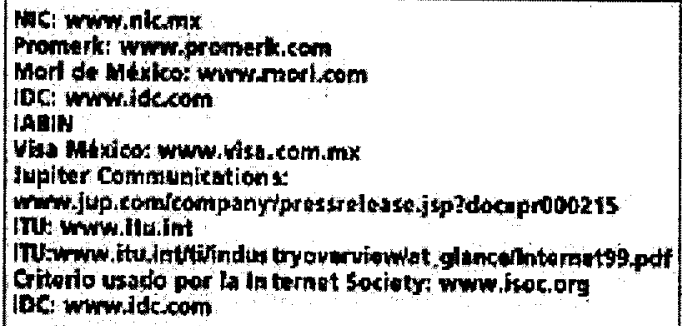 & 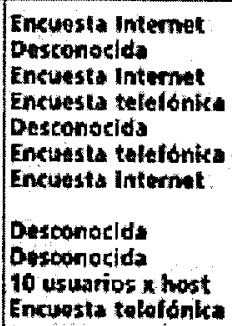 \\
\hline
\end{tabular}

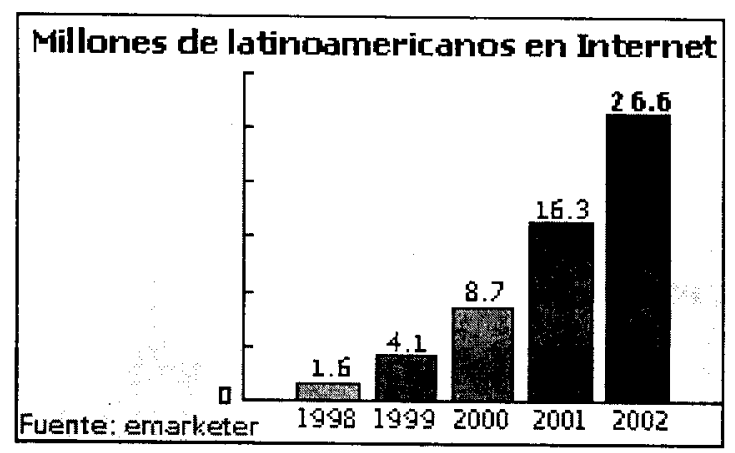

En México, se calcula que de los 100 millones de mexicanos, sólo el 5 por ciento posee una computadora personal, y sólo el 1.7 por ciento está en línea (Gómez Mont, 2000, en línea). Brasil, México y Chile tienen las redes más grandes en América Latina. Colombia y Panamá se cuentan entre las de crecimiento más rápido. El crecimiento mayor entre los países de habla castellana se ha dado en México [150\%], Colombia [125\%], Argentina [110\%] y Chile [71\%].

Un análisis de Baquía Inteligencia ${ }^{8}$ señala que hay motivos para ser optimistas, basando este optimismo en 3 preceptos:

6. http://www.etcetera.com.mx/pag59ne6.asp

7. http://www.pragma.com.co/pragmagazin/1999-10/cifrasLatinoPrint.html

8. http://www.baquia.com/com/legacy/14388.html 
- Latinoamérica cuenta con el gran valor de la juventud. La población latinoamericana se sitúa entre las más jóvenes del mundo. En el segmento entre los 10 y los 44 años, que comprende la mayoría de los usuarios de Internet, se concentra el $58,7 \%$ de la población, siete puntos más que en los Estados Unidos, y dos puntos más que la media mundial.

- Hasta el año 2003, la tasa de crecimiento de Internet en Latinoamérica será de un $47 \%$ anual, el ritmo más rápido en todo el mundo. En el año 2003 serán algo más de 19 millones los internautas latinoamericanos, lo que representará un 5\% de los 372 millones de usuarios en todo el mundo.

- Se calcula que existen unos 18 millones de PC en la región, lo que representa una penetración del 3,5\%. La mayoría se concentra en México (26\%) y Brasil (32\%). Se espera que de aquí al año 2003 el ritmo de crecimiento de esta tasa sea del $16 \%$ anual, más del doble del esperado en los Estados Unidos (7,3\%).

Un dato que es básico en la infraestructura de acceso al Internet es el del acceso telefónico, ya que "las posibilidades de crecimiento de Internet están estrechamente relacionadas con la calidad de líneas telefónicas en cada país y con los niveles de digitalización de las mismas" (Gómez Mont, 2000). Esto es debido a que la mayoría de conexiones se hace, desde hogares, vía telefónica. Para junio del 2000, la Cofetel daba la cifra de 11,640 líneas telefónicas en México. Sin embargo, las compañías que ofrecen acceso a Internet pueden continuar la tradición de medios en México de prácticas oligopólicas, ya que:

El grupo de telecomunicaciones Telmex lidera claramente el sector de las infraestructuras de acceso, gracias a su presencia en todo el territorio nacional y a su posición de dominio en los servicios telefónicos. Esta situación ventajosa le permite acaparar entre el 60 y el $65 \%$ de los accesos a Internet, situación que no ofrece visos de cambiar a corto plazo. Terra Networks ( $11 \%$ de cuota de mercado), PSINet (10\%) e InfoAccess (5\%) son los otros principales actores en el mercado de accesos a Internet.

La siguiente tabla ${ }^{9}$ ejemplifica la situación de México en comparación con otros países en cuanto al acceso a líneas telefónicas y en cuanto al acceso a Internet.

9. Tabla tomada de "Ciberdensidad en México: una comparación mundial" http://www.inegi.gob.mx/informatica/espanol/finformatica.html 


\begin{tabular}{|c|c|c|c|c|c|c|}
\hline & \multicolumn{2}{|c|}{ PIB per cápita } & \multicolumn{2}{|c|}{ Teledensidad } & \multicolumn{2}{|r|}{ Ciberdensidad } \\
\hline & $\begin{array}{l}\text { Número } \\
\text { de } \\
\text { paises }\end{array}$ & \begin{tabular}{|l} 
Promedio \\
(US\$)
\end{tabular} & $\begin{array}{l}\text { Número } \\
\text { de } \\
\text { paises }\end{array}$ & \begin{tabular}{|l|} 
Promedio \\
(líneas \\
telefónicas \\
por cada 100 \\
habitantes)
\end{tabular} & \begin{tabular}{|l|} 
Número \\
de \\
países
\end{tabular} & $\begin{array}{l}\text { Promedio (servidores } \\
\text { de Internet por cada } \\
\text { millón de habitantes) }\end{array}$ \\
\hline $\begin{array}{l}\text { Paises con } \\
\text { indicadores } \\
\text { mayores que los } \\
\text { mexicanos }\end{array}$ & 55 & $\$ 15,097$ & 71 & 32.12 & 31 & : 8,873 \\
\hline $\begin{array}{l}\text { Paises con } \\
\text { indicadores } \\
\text { menores que } \\
\text { los mexicanos }\end{array}$ & 118 & $\$ 1,070$ & 102 & 2.59 & 142 & 66.81 \\
\hline México & & $\$ 3,320$ & & 9.6 & & 973 \\
\hline
\end{tabular}

Reyes (1999) muestra en esta gráfica que:

La infraestructura telefóni ca no corresponde al ingreso de los mexicanos, que se encuentr a en el lugar 56. Sin embargo, al observar la posición de ciberdensidad nos encontramos en la posición 32; es decir, la relativamente baja teledensidad no ha impedido el desarrollo de Internet. Esto puede deberse a diversos factores, entre los que se encuentran un interés especial al respecto, la cercanía con los Estados Unidos o una mala distribu ción de la riqueza, lo cual implica que los indicadores de teledensidad y ciberdensidad se estén refiriendo solamente al sector de la población con ingresos más elevados (en línea).

Gómez Mont apuntala la situación de dependencia con los Estados Unidos al decir que

El Tratado de Libre Comercio con América del Norte ha sido el principal vector para el despegue de la infraestructura de la tecnología de Información y Comunicación. Este sector creció durante los últimos diez años cinco veces más que el resto de la economía mexicana, sin embargo, el nivel de desarrollo de la infraestructura informativa en México es aún muy bajo. (En líne a).

Hay solamente pistas, pero es imprescindible dar seguimiento a la evolución que vaya habiendo y poner el tema en la agenda política y social. 


\section{Comercio electrónico, acceso igualitario: el ejemplo de los artesanos en Internet}

Pero este vertiginoso camino hacia la conectividad tiene su base (como casi todos los desarrollos globales) en los procesos económicos que alientan dichas transformaciones. En la era en la que Amazon.com, una librería sin un solo libro, es la más importante del mundo, no hay más remedio que aceptar que el comercio electrónico es el futuro tecnológico de las transacciones comerciales. Nuevamente es vital una reflexión, en este caso sobre el acceso a las herramientas que hacen posible el desarrollo de un negocio en la llamada "Era Digital", que Negroponte (1995) anunciara con bombo y platillo y que otros tecnofílicos (extrañamente dueños de empresas o jefes de centros de cómputo) han adoptado secundando a Gates (1995), Detrouzous (1997), y a quienes hablan de un mundo democratizado, igualitario y moderno basado en el uso de tecnologías de información. Es una reflexión necesaria porque es obvio que "la tecnología de la información se convierte en la infraestructura primaria de toda la producción industrial y la distribución de bienes y servicios" (Hamelink, 1991, p. 16).

Un estudio de Baquía señala que "el valor del comercio electrónico en Latinoamérica será en 2004 de 82.000 millones de dólares. El 93\% de esta cifra corresponderá al negocio entre empresas". No podemos dejar pasar este dato, ya que las empresas que están en ventaja por su infraestructura y capital seguirán proponiendo las reglas del juego.

Ejemplos de cómo se han ido desplazando las instituciones comerciales a este tipo de economía pueden haber miles, tantos como giros haya en el comercio. Un ejemplo que proponemos más al azar que buscado, aunque podría ser cualquier otro, es el de los artesanos y su posible vinculación con el comercio electrónico. Lo escogimos porque contiene elementos que lo hacen interesante: una conexión lógica con las culturas regionales, prácticas y oficios ancestrales, y preguntas sobre el desarrollo de las pequeñas comunidades, siendo precisamente éstos algunos de los sectores más desprotegidos y recurridos en la discusión sobre la continuación de un proyecto globalizador que pueda ayudar a acortar la brecha entre los del sur y los del norte, entre los pobres y los ricos.

La reflexión lógica es que el comercio electrónico ha sido y es uno de los ejes básicos en esta evolución tecnológica y su clave es el Internet, en la que también el arte o la artesanía han encontrado un nicho importante. Utilizando el buscador Google ${ }^{10}$, que es la nueva generación de buscadores con mayor potencia y capacidad, al buscar la palabra "arte" se encontraron $3,350,000$

10. Búsqueda realizada el 27 de mayo de 2001 a las 21 horas. 
sitios (es digno de mencionarse el hecho de que el idioma es otro de los territorios golpeados en este "desarrollo", pues al buscar la palabra "art" se hallaron 40,200,000 sitios), mientras que al buscar la palabra "artesanía" se desplegaron 83,600 sitios; un dato interesante es que para la palabra "handcrafts" hubo apenas 31,500. Esto es un aliciente (o una preocupación según se vea), ya que hay más páginas en español que en inglés. Esto lo señala Gómez Mont (2000) como un detonante para el acceso a Internet en América Latina y apunta que este:

Ha tenido un crecimiento entre 1995 y 2000 del $800 \%$. Esta cifra será superada en unos cuantos meses, al incrementarse el materi al en español que circula en este nuevo medio. Hasta el año 2003, la tasa de crecimiento de Internet en Latinoamérica será de un $47 \%$ anual, el ritmo más rápido en todo el mundo. En el año 2003 serán algo más de 19 millones los internautas latinoamericanos, lo que representará un 5\% de los 372 millones de usuarios en todo el mundo. Se calcula que existen unos 18 millones de PC en la región, lo que representa una penetración del 3,5\%. La mayoría se concentran en México (26\%) y Brasil (32\%). Se espera que de aquí al año 2003, el ritmo de crecimiento de esta tasa sea del $16 \%$ anual, más del doble del esperado e los Estados Unidos (7,3\%).

Si bien es cierto que de ninguna manera podemos comparar estas cifras con los $81,700,000$ sitios detallados con la palabra "business", es importante mostrar que se han realizado esfuerzos por hacer de la red un espacio en donde quepan todas las propuestas. El ciberespacio es el centro comercial más grande del mundo, de manera que los artistas y artesanos regionales tendrán alguna oportunidad de competir en los mercados internacionales con șus productos siempre y cuando pueda desarrollarse una política de proteccionismo y equipamiento a aquellos productores $o$ asociaciones independientes para que entren al juego de la globalización, si no en las mejores condiciones, por lo menos con la capacidad para jugar ${ }^{11}$. Como apunta Casares (1991) "para adaptarnos a la tecnología, es necesario tomar como punto de partida las necesidades económicas, políticas y culturales, las de desarrollo en general, y las necesidades educativas en particular, para definir la manera de apropiación de la informática." (p. 230)

Experiencias en este sentido ya existen, y con éxito. En la serie de documentales sobre oficios tradicionales en pueblos de América Latina "Vidas Enraizadas", producida por la Universidad de Colima, se muestran dos; la de una cooperativa de textiles llamada Punha (que significa "Por un nuevo hombre americano", nombre muy ilustrativo), en Ambrapapma, Argentina, que mediante el uso del correo electrónico ha encontrado clientes en Europa

11. Es muy interesante el documento "Las tecnologías de la información y la comunicación en el desarrollo: reflexiones de la UNESCO" elaborado en 1996 y que vale la pena revisar. 
y Estados Unidos para sus productos; y el de un colectivo de mujeres guatemaltecas (uno de cuyos grupos se llama "catoquip" que significa "ayudémonos", otro nombre ilustrativo) que vende en Estados Unidos sus telas y productos terminados y que con la ayuda de una norteamericana tiene un canal de distribución de sus productos que próximamente estará en la red. Otro ejemplo ya consolidado es el de un grupo de artesanos de Cuzco, Perú que con la ayuda de la Universidad Nacional San Antonio Abad tiene venta de productos artesanales por Internet. ${ }^{12}$ Esto será sumamente importante, ya que de otra manera los grandes mercados absorberán la distribución y con ello las ganancias, dejando a los productores rezagados en este tipo de comercio.

La globalización económica y cultural es inevitable, por lo que debemos estar listos para interpretar los tiempos y proponer, secundar y apoyar propuestas artísticas o de difusión del arte que, mediante el uso correcto de la herramienta que representa las tecnologías, logren consolidarse y generar interés en ellas.

\section{A manera de conclusiones}

Parece ser que la informatización será la vía para la continuidad de un impulso globalizador y moderno en este principio de siglo. Como cualquier innovación, las llamadas nuevas tecnologías de la información y la comunicación tienen la capacidad de transformar la forma en la que la sociedad se organiza y evoluciona. Los países latinoamericanos tienen el gran reto de construir, desde una realidad que tome en cuenta las desigualdades, las culturas propias de cada región y las problemáticas nacionales, un proyecto que permita a la brevedad el desarrollo de una infraestructura informática que los convierta en partícipes en la construcción de una sociedad verdaderamente global y que posibilite la competencia en el mercado mundial de significados.

El Internet se convierte en el elemento clave en este proceso y tendrá la posibilidad de ser medio y fin: desde transacciones comerciales simples hasta controles macroeconómicos; desde instrumentos de poder y control que pueden ser usados para incrementar las desigualdades a herramientas liberadoras para lograr una sociedad más justa y democrática. Es fundamental la reflexión constante y el seguimiento empírico de los procesos y mecanismos que se gestan alrededor de ella, no sólo en el contenido de los mensajes, sino en el acceso a la posibilidad de consultarlos, usarlos y crearlos. 


\section{Bibliografía}

Arizpe, L. (1995). Las ciencias sociales y la creación de una nueva ética social. En Ruy Pérez Tamayo y Enrique Florescano (coord.) Sociedad, ciencia y cultura. México: Cal y Arena.

Barroso, P. (1998). Ética y Deontología de las nuevas tecnologías de la información y la comunicación (Internet). Revista Iberoamericana de Derecho de la Información 1. UIA: México.

Brand, S. (1988). The Media Lab. EUA: Penguin Books.

Casares, P. (1991). Informática, educación y dependencia. Necesidad de investigación y apropiación. En Carmen Gómez Mont (ed.) Nuevas Tecnologías de Comunicación. México: Trillas.

Cebrián, J. L. (1998). La Red. Madrid: Taurus.

Dertouzos, M. (1997). Qué será. México: Planeta.

Gubern, R. (1991). La Antropotrónica: Nuevos modelos tecnoculturales de la sociedad massmediática. En Carmen Gómez Mont (ed.) Nuevas Tecnologías de Comunicación. México: Trillas.

Hamelink, C. (1991). Informatización: Hacia una cultura binaria. En Carmen Gómez Mont (ed.) Nuevas Tecnologías de Comunicación. México: Trillas.

Mosco, V. (1989). The pay-per society: Computers and communication in the information age: essays in critical theory and public policy. EUA: Ablex.

Negroponte, N. (1995). Ser Digital. México: Océano.

Spínola, D. (1995). Los escenarios tecnológicos y el derecho a la información. En E. Villanueva (Coord.). Derecho y ética de la información. Media Comunicación: México

Straubhaar, J., y La Roce, R. (1996). Communications Media in the Information Society. Estados Unidos: Wasdworth Publishing Company.

Villanueva, E. (1995). Derecho a la información en una sociedad moderna y democrática. En E. Villanueva (Coord.). Derecho y ética de la información. Media Comunicación: México.

Reportes de Internet:

Por qué Latinoamérica sigue rezagada en la carrera de Internet (2000, junio).

Disponible en http://www.baquia.com/com/legacy/14388.html

Gómez Mont, C. (2000). La liberalización de las telecomunicaciones en México en el marco del TLCAN. En Razón y Palabra (en línea).

http://www.cem.itesm.mx/dacs/publicaciones/logos/actual/19_cgom ez.html Reyes, C. (1999). Ciberdensidad en México: una comparación mundial. En Boletín de Política Informática 4. En línea: http://www.inegi.gob.mx/informatica/espanol/finformatica.html 